\title{
Development of a Liquid-Junction/Low-Flow Interface for Phosphate Buffer Capillary Electrophoresis Mass Spectrometry
}

\author{
Fu-An Li, Ju-Li Huang, Shang-Yu Shen, Che-Wei Wang, and Guor-Rong Her* \\ Department of Chemistry, National Taiwan University, Taipei, Taiwan
}

To alleviate ion suppression from phosphate buffer and to preserve separation integrity, a new capillary electrophoresis mass spectrometry (CE-MS) interface was developed. The interface consisted of a low-flow interface and a liquid junction. In this design, both the inlet reservoir and the liquid-junction reservoir were filled with phosphate running buffer. Because the phosphate anions in the column migrated toward the inlet reservoir (away from the electrospray ionization (ESI) source) the problem of ion suppression in ESI was avoided. The liquid junction was incorporated to eliminate issues of degraded separation observed when sheath liquid interfaces use different buffers for separation and MS analysis attributed to differences in anion velocity. The utility of the interface was demonstrated by the analysis of antihistamines at $\mathrm{pH}$ 3.5 and the analysis of perfluorocarboxylic acid at pH 9.5.

Capillary electrophoresis (CE) is recognized as a highperformance separation technique that can be applied to a variety of molecules, including DNA fragments, proteins, peptides, and drugs. ${ }^{1-3}$ Mass spectrometry (MS) is arguably the most powerful detection method for CE. CE-MS, with its high separation efficiency and high compound identification capability, is an extremely valuable analytical technique for the analysis of complex mixtures. ${ }^{3-6}$

Among various background electrolytes (BGEs), phosphate buffer is one of the most commonly used BGE in the contemporary practice of capillary zone electrophoresis (CZE). Phosphate was the original buffer used by Jorgenson and Lukacs ${ }^{7,8}$ in their pioneering 1981 work that launched CZE to become a widespread modern analytical method. The overall experience with this easy to prepare and easy to use buffer is excellent. Therefore, it is generally the BGE of choice when starting to solve a new separation problem. Phosphate buffer has more than one useful

* To whom correspondence should be addressed. Phone: (886) 2-33661647. Fax: (886) 2-23638058. E-mail: grher@ntu.edu.tw.

(1) Dolník, V. Electrophoresis 2008, 29, 143-156.

(2) Kasicka, V. Electrophoresis 2008, 29, 179-206.

(3) Font, G.; Ruiz, M. J.; Fernández, M.; Picó, Y. Electrophoresis 2008, 29, 2059-2078.

(4) Simpson, D. C.; Smith, R. D. Electrophoresis 2005, 26, 1291-1305.

(5) Gaspar, A.; Englmann, M.; Fekete, A.; Harir, M.; Schmitt-Kopplin, P. Electrophoresis 2008, 29, 66-79.

(6) Kostal, V.; Katzenmeyer, J.; Arriaga, E. A. Anal. Chem. 2008, 80, 45334550 .

(7) Jorgenson, J. W.; Lukacs, K. D. A. Anal. Chem. 1981, 53, 1298-1302.

(8) Jorgenson, J. W.; Lucas, K. D. Science 1983, 222, 266-272. $\mathrm{p} K_{\mathrm{a}}$ and thus can be used over a wide $\mathrm{pH}$ range. Indeed, phosphate buffer often provides good peak shape and high separation efficiency. ${ }^{9}$ Although phosphate is a popular buffer for CE-UV applications, transferring methods developed for $\mathrm{CE}$ with UV detection to $\mathrm{CE}$ with electrospray ionization mass spectrometry (ESI-MS) detection is not an easy task, because it is known that phosphate buffer greatly suppresses analyte signal and contaminates the ion optics of the mass spectrometer. ${ }^{10}$ In addition, highly abundant phosphate cluster ions in the low-mass range have a severe negative impact on the ESIMS sensitivity of low-mass analytes. ${ }^{11}$ To alleviate these problems, volatile buffers such as ammonium acetate are often used to replace the nonvolatile phosphate buffers in mass spectrometric applications. ${ }^{12,13}$ Unfortunately, CE separation efficiency is not always maintained when phosphate buffer is replaced with a volatile buffer such as ammonium acetate. Thus, to extend the applicability of $\mathrm{CE}-\mathrm{MS}$, it is important to design an interface that removes phosphate without adversely affecting CE separation performance.

In order to alleviate the negative effect of BGE during the ionization process, an alternative could be the use of other ionization methods such as atmospheric pressure chemical ionization (APCI) or atmospheric pressure photoionization (APPI). Several attempts were reported for CE-APCI-MS hyphenation, ${ }^{14-17}$ but all resulted in poor sensitivity because most of APCI interfaces are designed for liquid chromatography mass spectrometry (LC-MS) at relatively high flow rates $(>100 \mu \mathrm{L} / \mathrm{min})$. APPI has been successfully applied both to CZE-MS ${ }^{18-22}$ and micellar electrokinetic chromatography mass spectrometry

(9) Gebauer, P.; Bocek, P. Electrophoresis 2000, 21, 2809-2813.

(10) Ju, D. D.; Lai, C. C.; Her, G. R. J. Chromatogr., A 1997, 779, 195-203.

(11) Juraschek, R.; Dulcks, T.; Karas, M. J. Am. Soc. Mass Spectrom. 1999, 10, 300-308.

(12) Kamel, A. M.; Brown, P. R.; Munson, B. Anal. Chem. 1999, 71, 968-977.

(13) Hiraoka, K.; Asakawa, Y.; Kawashima, Y.; Okazaki, S.; Nakamura, M.; Yamamoto, Y.; Takamizawa, A. Rapid Commun. Mass Spectrom. 2004, 18, 2437-2442.

(14) Tanaka, Y.; Otsuka, K.; Terabe, S. J. Pharm. Biomed. Anal. 2003, 30, 18891895.

(15) Takeda, S.; Tanaka, Y.; Yamane, M.; Siroma, Z.; Wakida, S.; Otsuka, K.; Terabe, S. J. Chromatogr., A 2001, 924, 415-420.

(16) Takada, Y.; Sakairi, M.; Koizumi, H. Anal. Chem. 1995, 67, 1474-1476.

(17) Isoo, K.; Otsuka, K.; Terabe, S. Electrophoresis 2001, 22, 3426-3432.

(18) Mol, R.; de Jong, G. J.; Somsen, G. W. Electrophoresis 2005, 26, 146-154.

(19) Nilsson, S. L.; Andersson, C.; Sjoberg, P. J. R.; Bylund, D.; Petersson, P.; Jomten-Karlsson, M.; Markides, K. E. Rapid Commun. Mass Spectrom. 2003, 17, 2267-2272.

(20) Hommerson, P.; Khan, A. M.; Bristow, T.; Niessen, W.; de Jong, G. J.; Somsen, G. W. Anal. Chem. 2007, 79, 5351-5357. 
(MEKC-MS) ${ }^{23,24}$ However, considering the complementary roles of APPI and ESI as well as the much wider availability of ESI, it is still important to improve the performance of CE-ESI-MS when a nonvolatile buffer is used for CE separation.

In CE-MS using phosphate buffer, ${ }^{25}$ one approach to alleviate the ion suppression is to control the $\mathrm{CE}$ conditions so that phosphate ions migrate toward the inlet reservoir instead of the ESI source, while replacing the sodium counterions with the more volatile ammonium ion. However, it was observed that the separation efficiency was degraded when the phosphate ions flowed away from the separation column. The degradation of separation integrity is most likely due to the liquid sheath effect, ${ }^{26}$ attributed to anions introduced into the column from the sheath liquid having a lower mobility than the phosphate anion.

In this study, a novel interface, liquid-junction/low-flow interface, was designed to alleviate the ion suppression effect of phosphate buffer. The interface consisted of a low-sheath-flow ESI interface and a liquid junction. For CE-MS operation, both the inlet reservoir and the liquid-junction reservoir were filled with the phosphate running buffer. In this design, as phosphate anions migrated toward the inlet reservoir during $\mathrm{CE}$ separation, phosphate anions from the liquid-junction reservoir were continuously supplied to the CE separation column. As a result, CE separation integrity was preserved. Because this interface also incorporated a sheath liquid prior to ESI-MS, it was possible to control the buffer composition introduced for ESI. The utility of the approach was demonstrated with the use of phosphate buffer in $\mathrm{CE}-\mathrm{MS}$ analysis under high-pH and low-pH conditions.

\section{EXPERIMENTAL SECTION}

Chemical Reagents. All chemical standards, carbinoxamine, pheniramine, chlorpheniramine, brompheniramine, doxylamine, perfluoroheptanoic acid, perfluorooctanoic acid, perfluorononanoic acid, perfluorodecanoic acid, and perfluorododecanoic acid were purchased from Sigma-Aldrich Chemical Co. (St. Louis, MO). Hydrofluoric acid (48\%) and ammonium hydroxide (28-30\%) were also purchased from Sigma-Aldrich Chemical Co. Ammonium acetate, ammonium dihydrogen phosphate, methanol (HPLC grade), sodium hydroxide, acetic acid (glacial), and phosphoric acid (85\%) were purchased from J. T. Baker (Phillipsburg, NJ). Deionized water (Milli-Q water system, Millipore Inc., Bedford, MA) was used in the preparation of the buffer solution and sheath liquid.

The phosphate buffer for the analysis of antihistamines was $60 \mathrm{mM}$ phosphoric acid adjusted to $\mathrm{pH} 3.5$ using ammonium hydroxide. The phosphate buffer in the analysis of perfluorocarboxylic acids was $50 \mathrm{mM}$ ammonium phosphate in 40\% isopropyl alcohol (v/v) adjusted to $\mathrm{pH} 9.5$ using ammonium hydroxide. Two different sheath liquids were used in the CE-MS analysis of

(21) Schappler, J.; Guillarme, D.; Prat, J.; Veuthey, J. L.; Rudaz, S. Electrophoresis 2007, 28, 3078-3087.

(22) Schappler, J.; Guillarme, D.; Rudaz, S.; Veuthey, J. L. Electrophoresis 2008, 29, 11-19.

(23) Mol, R.; de Jong, G. J.; Somsen, G. W. Anal. Chem. 2005, 77, 5277-5282.

(24) Himmelsbach, M.; Haunschmidt, M.; Buchberger, W.; Klampfl, C. W. Anal. Chem. 2007, 79, 1564-1568.

(25) Chien, C. T.; Li, F. A.; Huang, J. L.; Her, G. R. Electrophoresis 2007, 28, 1454-1460.

(26) Foret, F.; Thompson, T. J.; Vouros, P.; Karger, B. L. Anal. Chem. 1994, $66,4450-4458$.
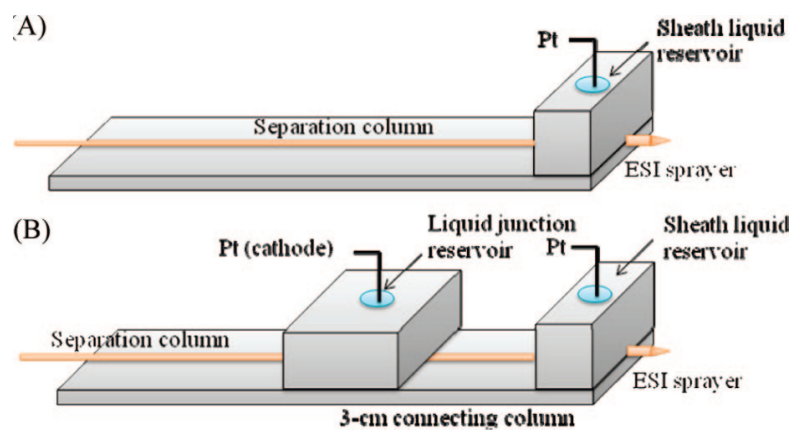

Figure 1. Schematic diagrams of $(A)$ the low-flow interface and $(B)$ the liquid-junction/low-flow interface. The ESI sprayer was tapered to $\sim 15 \mu \mathrm{m}$.

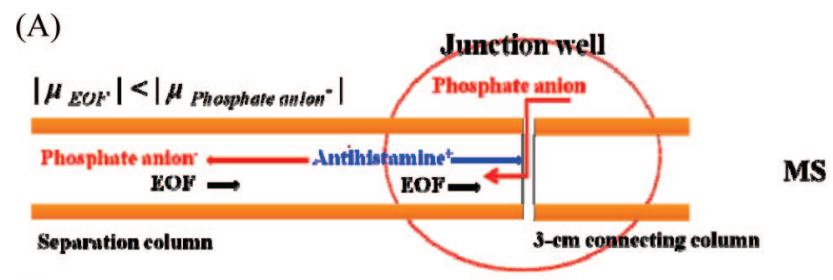

(B)

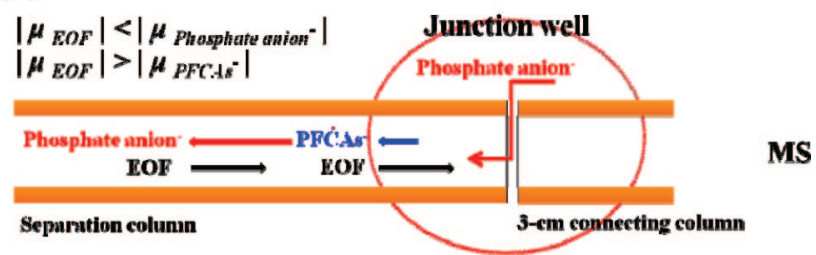

Figure 2. Schematic illustration of the migration behavior in the separation of $(A)$ antihistamines under an acidic condition and (B) PFCAs under a basic condition.

antihistamines: one was methanol-water-acetic acid (50:50:1, $\mathrm{v} / \mathrm{v} / \mathrm{v}$ ), and the other was methanol-water-phosphoric acid (50: $50: 0.01, \mathrm{v} / \mathrm{v} / \mathrm{v})$. The sheath liquid for the analysis of perfluorocarboxylic acids was $20 \mathrm{mM}$ ammonium acetate in 40\% isopropyl alcohol (v/v) adjusted to $\mathrm{pH} 9.5$ using ammonium hydroxide. Platinum wire was used as the electrode.

Low-Flow ESI Interface and Liquid-Junction/Low-Flow Interface. The low-flow ESI interface was fabricated as reported previously. ${ }^{27}$ As shown in Figure 1A, the low-flow interface consisted of a base and a low-flow sprayer.

The liquid-junction/low-flow interface is shown in Figure 1B. A liquid junction was added to the low-flow interface. ${ }^{27}$ The liquidjunction device was fabricated using poly(dimethylsiloxane) (PDMS) casting. The casting mold was assembled using poly (methyl methacrylate) (PMMA) materials (10 mm thickness). Four pieces of PMMA $(25 \mathrm{~mm} \times 10 \mathrm{~mm}$ and $35 \mathrm{~mm} \times 10 \mathrm{~mm}$, two pieces each) were fixed together on a PMMA plate $(50 \mathrm{~mm} \times 50$ $\mathrm{mm} \times 2 \mathrm{~mm}$ ) using $3 \mathrm{M}$ Scotch tape. Two holes (for holding a capillary column) were drilled on two PMMA plates (25 $\mathrm{mm} \times 10$ $\mathrm{mm}$ ) using a $400 \mu \mathrm{m}$ o.d. drill. A PMMA rod (20 mm length, 3 mm o.d.) was used to define the reservoir. A channel $(400 \mu \mathrm{m}$ i.d.) was drilled near one end of the rod. A capillary column (375 $\mu \mathrm{m}$ o.d.) was then inserted through the holes and the channel of the mold. The Sylgard 184 elastomers were mixed vigorously and degassed. The mixture was poured on the PMMA mold slowly

(27) Li, F. A.; Huang, J. L.; Her, G. R. Electrophoresis 2008, 29, 4938-4943. 
(A)

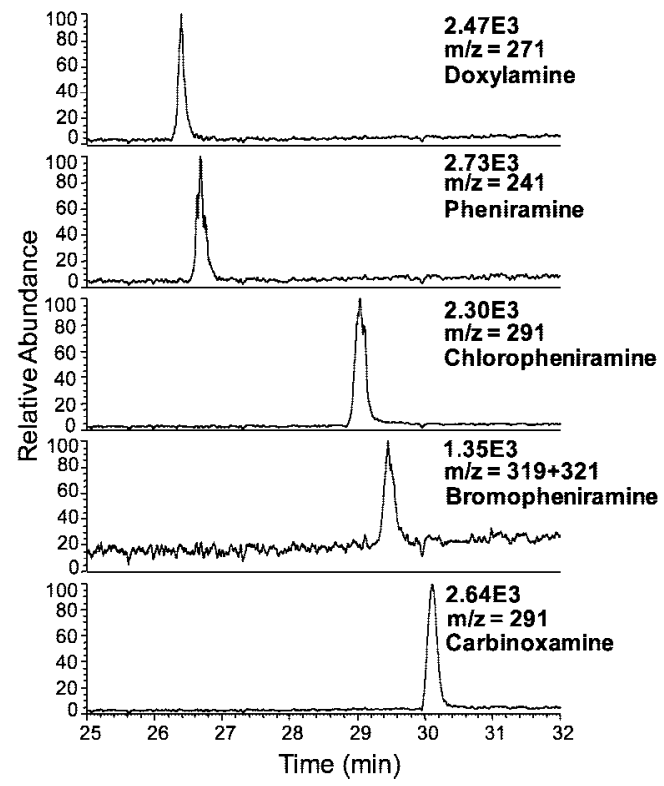

(B)

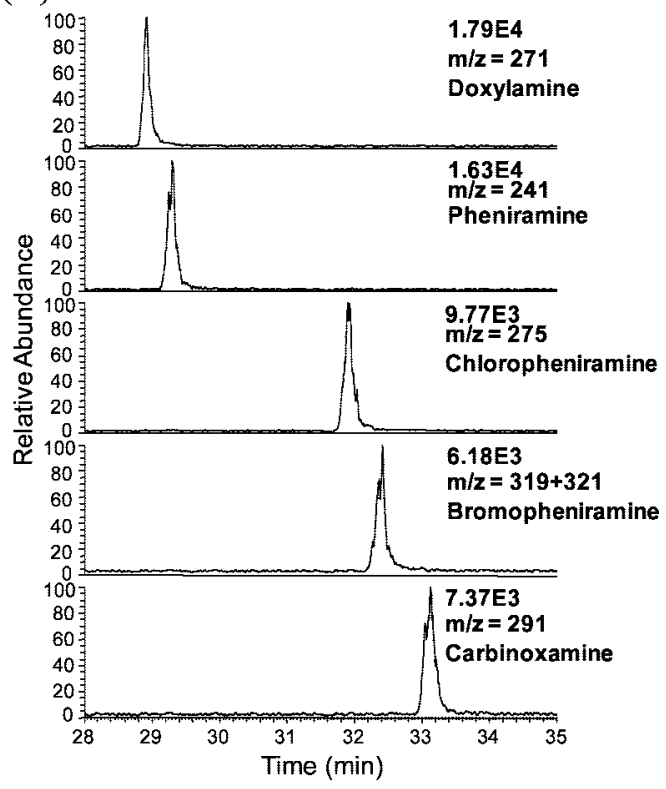

Figure 3. Extracted ion electropherograms of the analysis of an antihistamine mixture by CE-MS using phosphate buffer using (A) a low-flow CE-MS interface and (B) a liquid-junction/low-flow CE-MS interface.

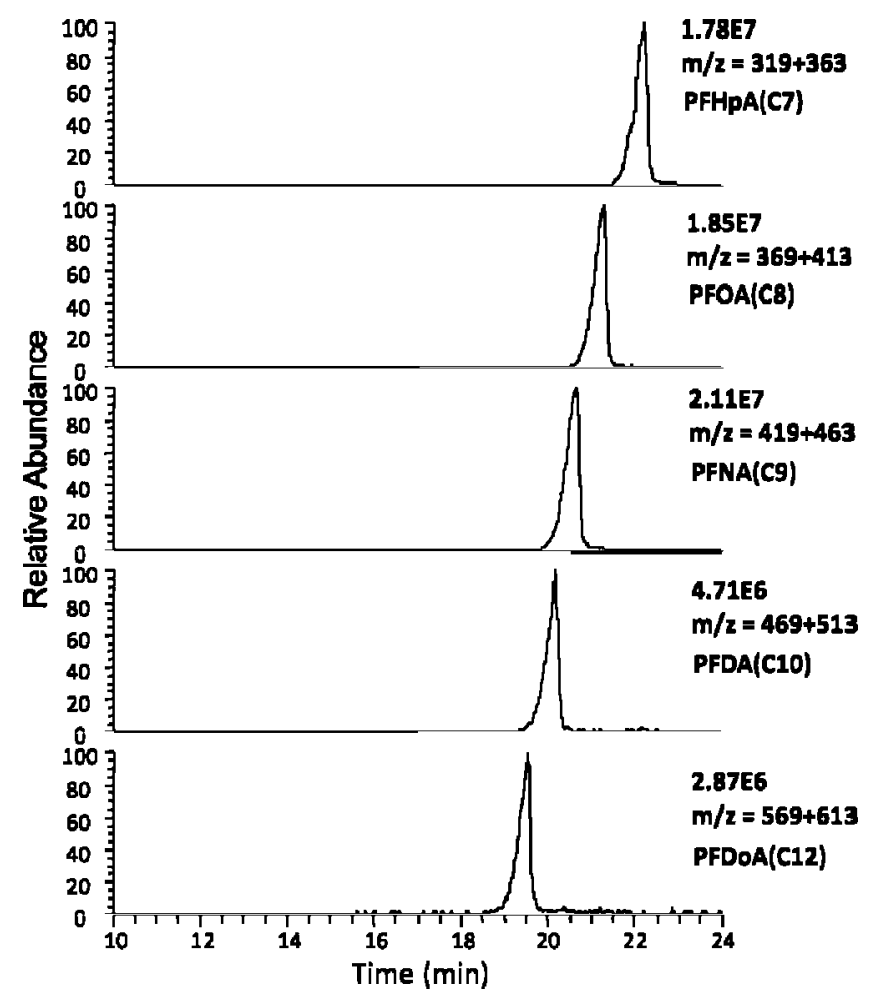

Figure 4. Extracted ion electropherograms of the analysis of PFCAs by $\mathrm{CE}-\mathrm{MS}$ using a liquid-junction/low-flow interface. Each trace represents the summation of the molecular ion and the fragment ion resulting from the loss of carbon dioxide from the molecular ion.

and cured at $70{ }^{\circ} \mathrm{C}$ for $4 \mathrm{~h}$ in a GC oven. After curing, the PMMA mold and the capillary were removed.

A $3 \mathrm{~cm}$ capillary (50 $\mu \mathrm{m}$ o.d., $375 \mu \mathrm{m}$ o.d., fused-silica capillary, Polymicro Technologies, Arizona) was used to connect the liquid junction to the low-flow interface. To reduce the dead volume, one end of the connecting capillary was tapered to $40 \mu \mathrm{m}$ before its insertion to the low-flow interface. The separation column (50 $\mu \mathrm{m}$ i.d. $\times 375 \mu \mathrm{m}$ o.d.) and the $3 \mathrm{~cm}$ connecting column $(50 \mu \mathrm{m}$ i.d. $\times 375 \mu \mathrm{m}$ o.d.) were inserted into the liquid junction to butt against each other. After inspecting the alignment under a microscope, the $40 \mu \mathrm{m}$ o.d. capillary end of the liquid-junction device was slid and inserted into the low-flow ESI interface and the device was ready for CE-MS analysis.

Mass Spectrometry. The analyses of histamine antagonists were performed on a LTQ linear ion trap (ThermoFinnigan, San Jose, $\mathrm{CA}$ ), and the instrument was operated in positive ion mode. The analysis of perfluorocarboxylic acids was conducted on an LCQ quadrupole ion trap (Finnigan MAT, San Jose, CA), and the instrument was operated in negative ion mode.

CE-MS Analysis of a Histamine Antagonists Mixture. In liquid-junction/low-flow CE-MS analysis of histamine antagonists, both the inlet reservoir and liquid-junction reservoir were filled with the phosphate running buffer $(60 \mathrm{mM}$ ammonium phosphate, $\mathrm{pH}$ 3.5). The acetic acid containing sheath liquid $\left(\mathrm{MeOH} / \mathrm{H}_{2} \mathrm{O} /\right.$ $\mathrm{CH}_{3} \mathrm{COOH}, 50 / 50 / 1(\mathrm{v} / \mathrm{v} / \mathrm{v})$ ) was added to the sheath liquid reservoir. Voltage was applied to the inlet buffer reservoir (anode) and the liquid-junction reservoir (cathode) by platinum electrodes using two high-voltage power supplies (CZE 1000 and CZE 2000, Spellman, Hauppauge, NY). The ESI voltage was supplied to the sheath liquid reservoir by the LTQ mass spectrometer. The sample solution (2 ppm antihistamine mixture) was hydrodynamically injected by raising the sample reservoir $10 \mathrm{~cm}$ for $15 \mathrm{~s}$. Separations were performed on a 90 $\mathrm{cm} \times 50 \mu \mathrm{m}$ i.d. $\times 365 \mu \mathrm{m}$ o.d. separation capillary, the effective electrical field of separation was set to $\sim 150 \mathrm{~V} / \mathrm{cm}$.

In low-flow $\mathrm{CE}-\mathrm{MS}$ analysis of histamine antagonists, the inlet reservoir was filled with phosphate running buffer and the sheath liquid reservoir was filled with $\mathrm{MeOH} / \mathrm{H}_{2} \mathrm{O} / \mathrm{H}_{3} \mathrm{PO}_{4}(50 / 50 / 0.01$, $\mathrm{v} / \mathrm{v} / \mathrm{v})$. The length of the separation column was $85-86 \mathrm{~cm}$ due to the fabrication of a tapered terminus. Other parameters were the same as liquid-junction/low-flow CE-MS analysis. 


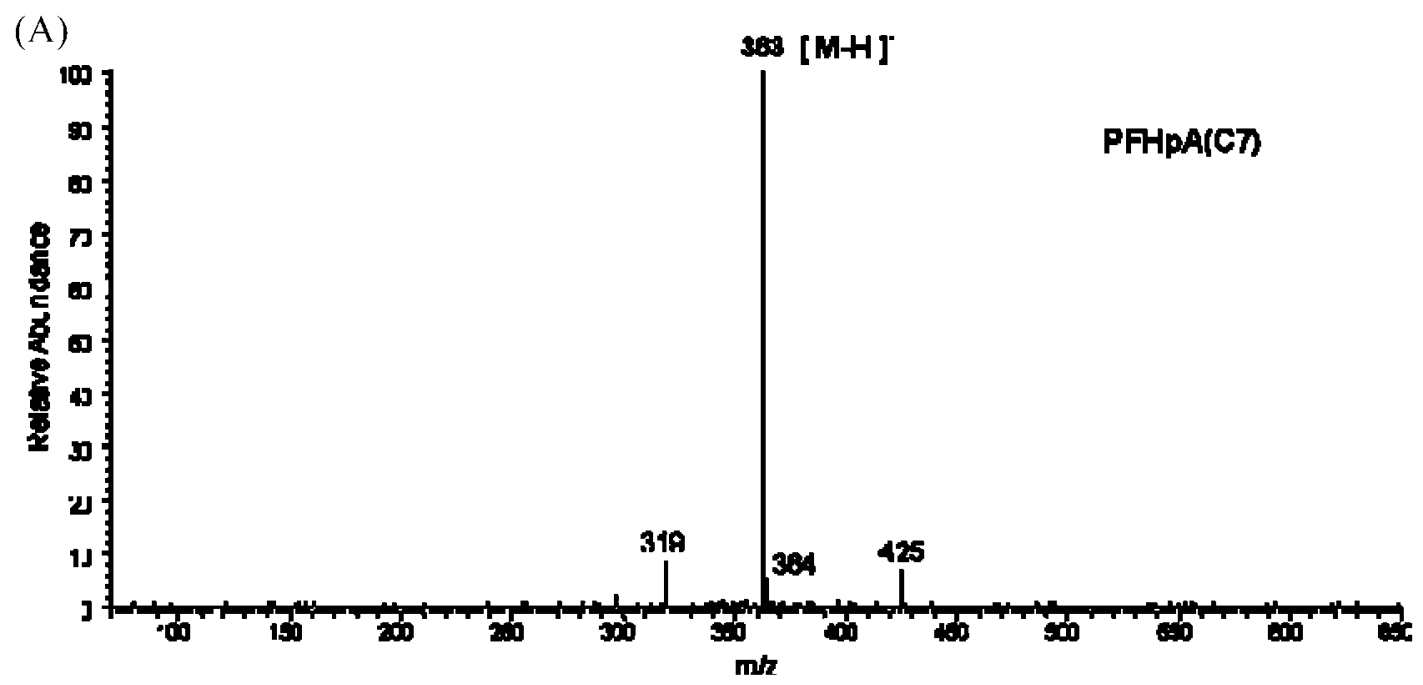

(B)

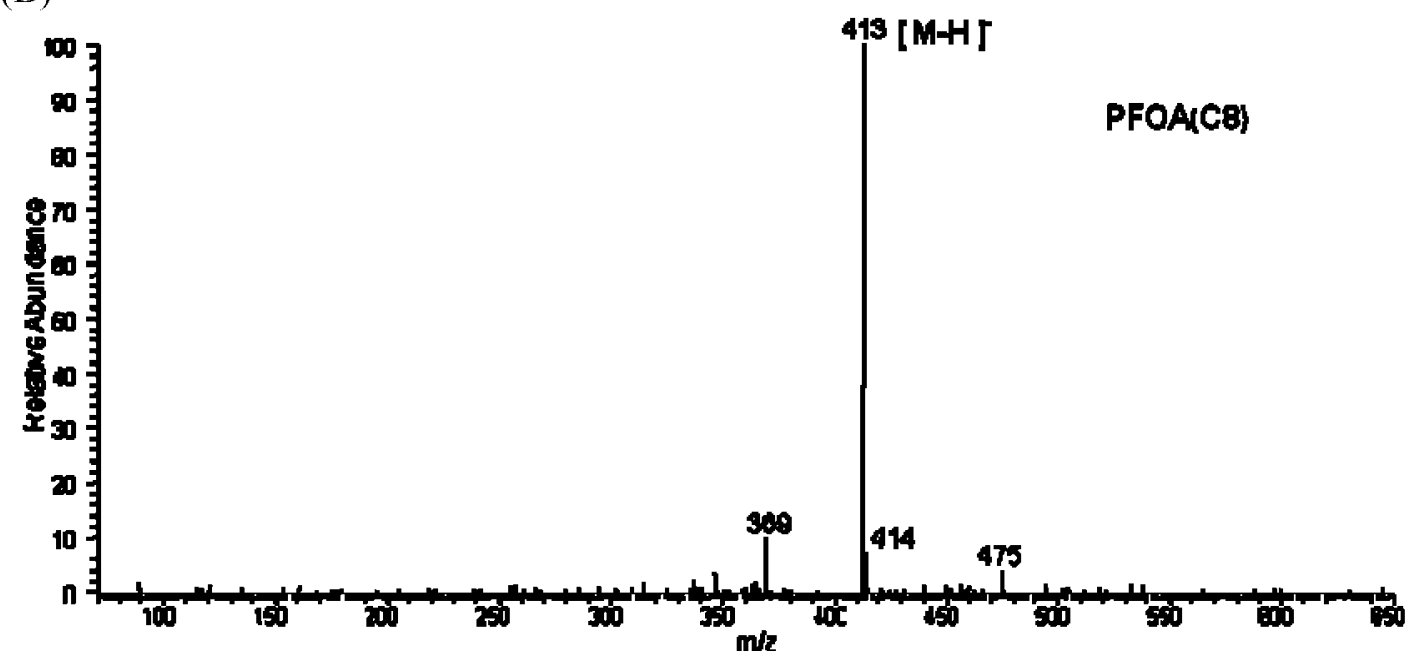

Figure 5. ESI mass spectra of the first two peaks in CE-MS analysis of PFCA: (A) the mass spectrum of perfluoroheptanoic acid (PFHpA) (C7); (B) the mass spectrum of perfluorooctanoic acid (PFOA) (C8).

CE-MS Analysis of Perfluorocarboxylic Acids. In CE-MS analysis of perfluorocarboxylic acids, both the inlet reservoir and liquid-junction reservoir were filled with the phosphate running buffer (50 mM ammonium phosphate, 40\% isopropyl alcohol, $\mathrm{pH}$ 9.5). The sheath liquid reservoir was filled with sheath liquid (20 $\mathrm{mM}$ ammonium acetate, $\mathrm{pH}$ 9.5). The sample solution (10 ppm perfluorocarboxylic acids mixture) was hydrodynamically injected by raising the sample reservoir $10 \mathrm{~cm}$ for $10 \mathrm{~s}$. Separations were performed on a $40 \mathrm{~cm} \times 50 \mu \mathrm{m}$ i.d. $\times 365 \mu \mathrm{m}$ o.d. separation capillary; the effective electrical field of separation was set to $\sim 400$ $\mathrm{V} / \mathrm{cm}$.

Safety Considerations. The $\mathrm{CE}$ high voltage should be used with caution.

\section{RESULTS AND DISCUSSION}

Liquid-Junction/Low-Flow Interface. The strategy used in this approach for alleviating ion suppression from phosphate buffer was to keep phosphate ions from entering the ESI source. This was achieved by controlling the mobility of phosphate ion and the electroosmotic flow (EOF) so that phosphate ions had a net mobility in the direction of the inlet reservoir. This strategy was previously implemented in a $\mathrm{CE}-\mathrm{MS}$ approach using a low-flow interface; however, the separation integrity was found to be degraded. ${ }^{25}$ In this approach, although phosphate ions migrated toward the inlet reservoir, anions from the sheath liquid reservoir entered the separation column. Because the anions entering the separation column from the sheath liquid (e.g., acetate) have a lower mobility than the phosphate, the separation integrity was degraded due to the liquid sheath effect. To circumvent the liquid sheath effect, a small amount of phosphoric acid was added in the sheath liquid. Although the liquid sheath effect was reduced, signal suppression caused by phosphate ions introduced from the sheath liquid was observed. ${ }^{25}$

In order to reduce both the liquid sheath effect and signal suppression, a new interface was proposed. A liquid-junction device was added to the low-flow interface as shown in Figure 1B. The liquid-junction reservoir served as the cathode for $\mathrm{CE}$ separation. The reservoir was filled with phosphate buffer to create a separation condition similar to a conventional CE-UV setup. In this design, while phosphate ions in the separation column migrate toward the inlet reservoir, phosphate ions in the liquid-junction reservoir are supplied continuously to the separation column so that CE separation integrity is preserved. 
A short connecting capillary (about $3 \mathrm{~cm}$ ) was added between the liquid-junction reservoir and the low-flow ESI interface to allow a connection for sheath liquid introduction. To reduce the dead volume between the separation column and the connecting capillary, the two columns were positioned in the center of the liquid-junction reservoir with a gap of less than $10 \mu \mathrm{m}$. As mentioned earlier, the liquid-junction device was fabricated using PDMS. One advantage of using PDMS as the building material is that the alignment of the separation column and the connecting column can be better monitored due to the transparency of the material. Furthermore, the elastomeric properties of PDMS can tightly seal the column to prevent the leakage of buffer solution.

The benefit of low sheath flow is preserved in this new approach. The low sheath flow provides the advantages of low sample dilution and the flexibility to modify the solution for better ESI sensitivity. In addition, unlike the situation encountered with a low-flow interface, no modification of the separation column is needed.

CE-MS Analysis of Histamine Antagonists. Under an acidic condition such as $\mathrm{pH} 3.5$, the phosphate ion has significantly higher mobility $\left(-3.41 \times 10^{-4} \mathrm{~cm}^{2} / \mathrm{V} \cdot \mathrm{s}\right)$ than EOF $\left(1.06 \times 10^{-4}\right.$ $\mathrm{cm}^{2} / \mathrm{V} \cdot \mathrm{s}$ ), and thus the phosphate anions would flow toward the inlet reservoir (anode) instead of to the ESI (cathode). At $\mathrm{pH} 3.5$, all the antihistamines possess positive charge and the direction of the mobility is the same as the EOF. Therefore, they will flow toward the ESI source. The migration behavior of the EOF, the analyte, and the phosphate ion is shown in Figure 2A. Antihistamines have been analyzed by $\mathrm{CE}-\mathrm{MS}$ using phosphate buffer and a low-flow CE-MS interface. ${ }^{25}$ For the purpose of comparison, a $2 \mathrm{ppm}$ antihistamine mixture was analyzed using both a low-flow interface and a liquid-junction/ low-flow interface. In the low-flow interface, to avoid the liquid sheath effect, $0.01 \%$ phosphoric acid was added to the sheath liquid. In the liquid-junction/low-flow interface, the $60 \mathrm{mM}$ phosphate running buffer was added to the inlet as well as the liquid-junction reservoir. The results reveled that the peak intensity using liquid-junction/low-flow interface (Figure 3B) was $\sim 3-7$ times higher than that obtained using a low-flow interface (Figure $3 \mathrm{~A})$. The improved signal was very likely due to the use of a sheath liquid without any phosphate ion.

One major concern of using a liquid junction in the new interface is the possibility of peak broadening. As can be seen in Figure 3, the peak widths at half-height of the two approaches were similar (low-flow interface, 7.0 to $\sim 9.5 \mathrm{~s}$; liquid-junction/ low-flow interface, 7.5 to $\sim 9.3 \mathrm{~s} ; N=3$ ).

CE-MS Analysis of Perfluorocarboxylic Acids. For acidic compounds such as perfluoroalkyl carboxylates (PFCAs), a negative ion would be a good choice for ESI detection. Therefore, a basic buffer is an appropriate condition for $\mathrm{CE}-\mathrm{MS}$ analysis of PFCAs. However, the mobility of the EOF under basic conditions is significantly higher than that at acidic $\mathrm{pH}$. Therefore, the EOF can carry the phosphate ion to the ESI source resulting in ion suppression.

To prevent phosphate ions from entering the ESI source, the factors affecting the EOF and electrophoretic mobility must be considered. One factor affecting the EOF in $\mathrm{CE}$ is the presence of organic modifiers in the BGE. Organic modifiers can decrease the EOF and the effective mobility of analytes. The organic modifier, isopropyl alcohol, has been used in the analysis of PFCAs by CE-UV. Wójcik et al. reported the use of a $50 \mathrm{mM}$ phosphate buffer with $40 \%$ isopropyl alcohol in the separation of long chain PFCAs. ${ }^{28}$ The same buffer composition was adopted in this $\mathrm{CE}-\mathrm{MS}$ approach. The migration behavior of the PFCAs, the phosphate ion, and the EOF is illustrated in Figure 2B. Both the PFCAs and the phosphate ions are flowing against the EOF. However, the phosphate ion has a higher mobility than EOF $(-2.3$ $\left.\times 10^{-4} \mathrm{vs} 1.7 \times 10^{-4} \mathrm{~cm}^{2} / \mathrm{V} \cdot \mathrm{s}\right)$, whereas the PFCAs have a lower mobility than EOF ( -7 to $\sim 9 \times 10^{-5}$ vs $\left.1.7 \times 10^{-4} \mathrm{~cm}^{2} / \mathrm{V} \cdot \mathrm{s}\right)$. As a result, the PFCAs will migrate toward the ESI source, but the phosphate anions will be carried in the opposite direction.

In the CE-MS analysis of PFCAs using a liquid-junction/lowflow interface, phosphate ions in the junction reservoir will be continuously supplied to the separation column, and thus the separation efficiency should be preserved. The extracted ion electropherograms (Figure 4) of a PFCAs mixture revealed that all five perfluorocarboxylic acids were separated and the ion suppression is alleviated as evidenced by the very good signalto-noise $(\mathrm{S} / \mathrm{N})$ ratios. Figure 5 contains the mass spectra from the first two PFCAs. As expected, ions related to phosphate buffer were not detected.

\section{CONCLUSIONS}

A liquid-junction/low-flow interface has been developed to facilitate the use of nonvolatile phosphate buffer in CE-MS applications. Capitalizing on differences in mobility between the EOF and the phosphate anions, the phosphate anions can be directed toward the inlet reservoir (away from the ESI source) to avoid ion suppression. A liquid-junction reservoir filled with phosphate running buffer was added to a low-flow interface. With this arrangement, the phosphate ions can be continuously supplied to the separation column to preserve the separation integrity. Since the concept of low-flow interface was adopted, the benefits of a low sheath flow such as a low dilution factor and the ability to modify the spray solution for higher ESI sensitivity was preserved. $\mathrm{CE}-\mathrm{MS}$ results from two applications provided evidence phosphaterelated ion suppression was successfully alleviated without degrading CE separation efficiency.

\section{ACKNOWLEDGMENT}

This work was supported by the National Research Council of the Republic of China.

Received for review November 25, 2008. Accepted February 1, 2009.

\section{AC802491Y}

(28) Wójcik, L.; Szostek, B.; Maruszak, W.; Trojanowicz, M. Electrophoresis 2005, 26, 1080-1088. 